\title{
GALAXIES
}

Commission 28 was involved in an all-day Joint Commission Meeting, of which the proceedings will be published in the report of Commission 29:

30 July: Early Nucleosynthesis in Galaxies (Commissions 28, 29, 35).

One business session and one scientific session on "New Results" were held, while four Working Groups each organized one or two half-day session(s). Short abstracts, where available, of the papers presented are given in the following.

\section{Julv: Business}

\section{CHAIR: G.A. Tammann}

The following items were covered in the business section:

Oraft report. The Commission adopted the report which was distributed to the members of the Commission and which has already appeared in Report on Astronomy vol. XXI A. The desirability of the reports is discussed. Of 40 members present, only 28 have read the report, but 38 members voted for a continuation of the reports.

Officers. The Commission unanimously elected E.Ye. Khachikian (USSR) as President for the following three years and V.L. Trimble (USA) as Vice-President. Outgoing members of the Organizing Committee are J. Lequeux (France), Li Qi-bin (China), H. Quintana (Chile), and P. van der Kruit (Netherlands). They are thanked for their in part very important services for the Commission. Continuing members are $\mathrm{F}$. Bertola (Italy), R.S. Ellis (UK), K.C. Freeman (Australia), J.S. Gallagher (USA), S. Okamura (Japan), and G.A. Tammann (Switzerland). The Commission elected as new members G. Bruzual (Venezuela), F. Israel (Netherlands), Jian-sheng Chen (China), and M.H. Ulrich (ESO). The Commission took note of a list of 95 new members who the President has accepted during his three-year term.

Resolutions. The Commission approved the resolution on World Astronomy Days as introduced by W. Wamsteker and the resolution on ASCHOT ( $80 \mathrm{~cm}$ space Schmidt) as introduced by H.C. Arp.

Working Groups. C.J. Peterson informed that the WG on "Internal Motions in Galaxies" and "Galaxy Photometry and Spectrophotometry" will be joint. W. Liller announced his wish to pass on his chair of the WG on "Supernovae". The WG on "Redshifts of Galaxies" under J. Huchra and on "Nomenclature" under K.S. de Boer had no sessions during the General Assembly.

Other business. The Commission was informed of forthcoming conferences on galaxies.

\section{July: New Results}

\section{CHAIR: G.A. Tammann}

Abstracts of the following papers were received:

\section{S. Djorgovski, Pasadena: Systematics of Galaxy Properties: Hints about their Formation.}

Observed ranges of galaxian global properties and the scaling laws and correlations between them reflect processes of galaxy formation and evolution, and thus contain interesting cosmological information. 
The basic correlations which tie most of the global properties of galaxies are intrinsically bivariate, both for ellipticals and spirals. The origins of these correlations are not yet fully understood, but some hints about galaxy formation can be deduced from them. Slight environmental variations, possibly caused by differences in the formation histories, may exist in correlations used as distance indicators, and appear as spurious peculiar velocities. Galaxies form two-dimensional sequences in the parameter space whose axes reflect the size (mass, luminosity, or radius), density or surface brightness, and kinetic temperature (velocity dispersion, circular speed for disks). This galaxy parameter space promises to become an equivalent of the H-R diagram for galaxies, a new organizing framework for extragalactic astronomy and cosmology.

\section{A.P. Fairall, Cape Town: A Southern Redshift Catalogue.}

An updated version of "Southern Redshifts - Catalogue and plots" (with A. Jones) will be available from October. It provides a "best-estimate" of the heliocentric radial velocities of almost 13000 galaxies south of Declination $0^{\circ}$. It is based on towards 17000 redshift measurements, from over 200 sources, either published or otherwise made public. Each entry is identified as optical, radio (or both) and carries an indication of optical emission lines where present.

Where two or more redshift measurements are available for the same galaxy, an assessment of redshift errors is possible. As a general rule, external errors are twice those claimed by authors, while approximately $1 \%$ of redshifts from each reference are erroneous. A significant number of discrepant velocities occur - many from recent fibre-optic spectroscopy.

Redshift plots - "slices" in R.A. and Declination can be generated from the catalogue; if required, various emission-line categories can be identified. These map structures in the southern skies, including superclusters, great and lesser walls, and numerous voids.

\section{V.G. Gurzadyan, Yerevan: The Dynamics of Galaxies and Clusters: The Concept of Ergodic Theory.}

The metrical theory of Dynamical Systems, Ergodic Theory enables one to investigate most essential topics of the dynamics of galaxies and clusters. Among the important ones is the result that relaxation of real stellar systems, globular clusters and elliptical galaxies occurs not due to binary encounters, as was considered for decades, but by means of collective interaction of all stars. The corresponding collective relaxation time is smaller than the binary one. This fact has numerous fundamental consequences, including the solution of Zwicky's paradox for elliptical galaxies. An interesting observational indication of this fact was obtained recently by Enrico Vesperini of the Scuola Normale Superiore of Pisa while investigating the core collapse phenomena for 127 globular clusters: the binary relaxation time scale does not fit the observational data while the formula for collective one does it quite well.

Disk stellar systems investigated by means of the properties of groups of diffeomorphisms on right-invariant Riemannian metrical spaces demonstrate different features: the motion is exponentially unstable, while the velocity field remains stable. The corresponding mixing time (not relaxation) for the Galaxy and Solar neighbourhood yields 100 million years.

The methods are very useful while investigating many non-trivial properties of the dynamics of other types of stellar systems (including those derived by means of numerical experiments).

\section{Slobodan Ninkovic, Zlatko Catovic: On the Potential in the Local Group of Galaxies.}

Assuming a rectilinear approaching of the two most massive Local Group galaxies, M31 and our Galaxy, the motion of a test particle in their field is examined. For the potential of an individual galaxy we assume the form $\left.G M / r^{2}+b^{2}\right)^{1 / 2} ; G$ - gravitation constant, $M$ - mass of the galaxy, $r$ - distance to its center, $b$ - constance. The examined cases indicate that even if masses of the Milky Way and of the Andromeda Galaxy are not too high (say about $5 \times 10^{11} \mathrm{M}_{0}$ ) and the masses are nearly equal, the apogalactic distances at a few hundreds kpc seem not to result in stable orbits. 
Slobodan Ninkovic, Zlatko Catovic: An Investigation of the Andromeda Galaxy.

Assuming the spherical symmetry with a constant circular velocity the present author studies the Andromeda Galaxy using a sample of 158 globular clusters. The conclusions are that the eccentricities of the clusters seem to be high and that their line-of-sight velocities suggests a value of about $175 \mathrm{~km} \mathrm{~s}^{-1}$ as a lower limit to the circular velocity.

\section{I.A. Issa, Cairo: Archimedes Spiral and the Surface Distribution of Dark Clouds in M31.}

The surface distribution of dark clouds along the major and minor axes were combined to give a general formula expressing the surface distribution along both axes simultaneously. Absorption values were determined, assuming the parameters of the standard cloud model as a function of the distance along both axes. Isocloud line numbers were drawn as a function of the distance along both axes. Two knots appeared in the new distribution function. This may announce the beginning of spiral arms. The knots may indicate the least possible numbers of dark clouds per kiloparsec for a spiral arm to appear.

M. Kalinkov and I. Kuneva, Sofia: Superclusters, Supervoids, and Minivoids among Rich Clusters of Galaxies.

Multivariate redshift estimates for AC0 clusters of galaxies with unknown redshift are made. A catalog of 196 superclusters is presented (density contrast 40 and percolation radius of $100 \mathrm{Mpc}$ ). We found 96 doublets of clusters, 10 supervoids and 39 minivoids among rich clusters of galaxies.

\section{Kuneva and M. Kalinkov, Sofia: Reference Catalog of ACo Clusters of Galaxies.}

The Reference Catalog of all ACO (Abell, Corwin, and Olowin, 1989) clusters of galaxies is completed. The Catalog contains optical, radio, and X-ray data, gathered from the literature. In fact many catalogs (Abell, AC0, Leir and van den Bergh, Owen et al., Struble and Rood, ...) are merged and some of the characteristics were reduced into a homogeneous system $\left(\mathrm{H}_{\mathrm{o}}=100 \mathrm{~km} \mathrm{~s}^{-1} \mathrm{Mpc}^{-1}\right.$ and $\left.\mathrm{q}_{\mathrm{o}}=1 / 2\right)$. The Catalog contains references for observations or theoretical investigations of the clusters. The references are given with their titles and key words.

\section{July: WG on Space Schmidt Survey}

\section{CHAIR: H. Arp}

The necessity for a wide-angle UV space telescope for astrophysical investigations was discussed. Some of the main scientific goals such as full sky survey, study of UV emission of extragalactic and galactic objects as well as faint-surface brightness research both in UV and near IR which may be achieved by the use of an orbital Schmidt-type telescope were presented.

As an introduction to the discussion the following paper was given: H. Lorenz (Germany): The Astrophysical Orbital Telescope ASCHOT. The project has been developed in the Bjurakan Astrophysical Observatory (Armenia) and the Central Institute for Astrophysics Potsdam (Germany). The optical system of the telescope is of fully reflecting Schmidt type. The diameters of the main spherical mirror and the correcting mirror are $1200 \mathrm{~mm}$ and $800 \mathrm{~mm}$, respectively. The focal length is $2300 \mathrm{~mm}$ and the field of view $5^{\circ}$. The displacement of the focal surface out of the telescope permits to use several detectors for different wavelength regions. The sensitivity of the telescope is estimated to be about $24^{\mathrm{m}}$ for stellar objects at $1500 \mathrm{~A}$.

The telescope will be launched in 1996. 
CHAIR: M. Feast

The following Organizing Committee was elected: Walborn (USA; Chairman), Wood (Australia), De Boer (Germany), Suntzeff (USA, Chile) and Feast (South Africa).

The following papers were read:

(A) HST Observations of the Magellanic Clouds.

N. Panagia: The SN 1987A Ring and the Distance of the LMC.

B. Campbell: Wide Field/Planetary Camera Images of the 30 Doradus Central Cluster.

S. Shore: $\quad$ Goddard High Resolution Spectrograph Observations of Melnick 42 and R136 in 30

Doradus.

N. Walborn: Spectral Morphology of Melnick 42 and Faint Object Camera Images of R136 (for G.

C. Blades: Faint Object Camera Images of Planetary Nebulae in the Magellanic Clouds.

(B) General

M. Bessell: Chemical Abundances of K Giants in the Magellanic Clouds.

D. Hatzidimitriou, R. Cannon and M. Hawkings: The Kinematics of intermediate-age and old stars in the outer parts of the SMC.

H. Dottori and E. Bica: Magellanic Cloud Clusters.

R. Wielebinski: The Status of SMC/LMC Radio Continuum Surveys.

A. Feinstein, R. Vazques and W. Seggewiss: The Young Open Clusters NGC 1962-65-66-70 in the LMC.

W. Seggewiss, K.S. de Boer, T. Richtler et al.: Comparison of Cluster and Field Star Populations in the Magellanic Clouds.

S. Shore: $\quad$ Studies of Novae in the Magellanic Clouds.

N. Martin, H. Lindgren and M.C. Lortet: Star Formation History in the 30 Doradus and Shapley II Constellation Areas.

R. Wing: Statistics of K supergiants in the LMC.

27 July: WG on Internal Motions in Galaxies and Galaxy Photometry and Spectrophotometry

CHAIR: C.J. Peterson

Abstracts of the following papers have been received.

\section{P. Pismis, Mexico: Annular Features in Spiral Galaxies Rather Spirals than Rings.}

Brightness enhanced annular structures within early type spiral galaxies particularly of the barred type are known to exist. These structures are referred to as "rings". In no case are they genuine rings but rather spirals. Bi-symmetrical details shown in well observed high resolution images attest clearly that the annular features consist of section of symmetrical spirals and are not torus-like structures.

To insist that the annular structures should be termed tight spirals and not rings is not a matter of semantics. To call such features "rings" disregards their bi-symmetrical nature, concealing thus the very property which is crucial in deciphering the origin of the so-called rings.

A classification of "rings" is currently accepted; it contains: rings galaxies, polar rings, ringed galaxies in which are defined outer, inner and nuclear "rings". In all cases they are not genuine rings as hinted above. We call attention in particular to nuclear annuli of early type, mostly barred galaxies. Nuclear "rings" are unmistakably tightly wrapped spirals. These show rotation around the nucleus as well as radial motions; the latter may be outward or inward.

In a series of papers (Pismis and Moreno 1984 and on) we have developed a model which explains the formation of tight nuclear spirals. The model: a) Matter (plasma) is ejected from localized areas on a galactic nucleus (ejection is thus neither isotropic nor toroidal). b) These areas are located in a bi-polar 
fashion on the equator of a rotating nucleus. c) Collimation of the outflow is ensured by magnetic lines of force which entrain the plasma.

The equation of the locus of the ejecta $t$ years after the onset of ejection is given by Pismis and Moreno $(1987,1990)$. We have applied our model to the central spiral of NGC 4736 adopting its physical parameters from van der Kruit (1976). The computed spiral agrees remarkably well with the observed kinematics as well as with the morphology. Also our model predicts aside from outward motions, which decrease along the opening of the spiral, also inward motions detected in some galaxies. These circumstances constitute a crucial test to our model.

As to energy requirements, to produce an expanding ring will require energy at least three orders of magnitude higher than the energy to produce an expanding tight spiral of similar dimensions.

We have undertaken multi-color photometric as well as spectrophotometric studies of the bulges of a selected group of early type spirals with the aim to determine the detailed morphology and the velocity structure of their nuclear spirals and thus be able to check further our model for the origin and development of the tightly wrapped nuclear spirals.

\section{T.K. Chatterjee, Mexico: The Frequency of Merging Galaxies and their Dynamical Implications.}

A study of the expected frequency of merging galaxies (not considering the galaxies to be embedded in massive halos), on the basis of the collisional theory of their formation, is conducted by studying collisions with different parameters and with different progenitor pairs. Assuming that $\sim 10 \%$ of all galaxies are ellipticals, our results indicate that $\sim 0.1 \%$ of all ellipticals are expected to be merger products. In the light of the current observational evidence, especially of the existence of a "fundamental parameter plane" where the global properties of ellipticals lie, a strong regularity in elliptical galaxy formation is indicated. The results favour the formation of ellipticals by dissipative collapse, followed by secular evolution by mergers.

\section{July: WG on Supernovae}

\section{CHAIR: V. Trimble}

Nine papers were scheduled and delivered in this session. Abstracts of three of them, provided by the authors, conclude this section. Highlights of the others include:

Radio light curves (N. Panagia). Five Type II events, and no Ia's, have now been seen. Radio light curves can be interpreted as ejecta shocking red giant winds of progenitors. Based on this interpretation, the progenitor of $1979 \mathrm{C}$ was at least $13 \mathrm{M}_{\mathrm{o}}$ and shed a wind of about $10^{-4} \mathrm{M}_{\mathrm{o}} / \mathrm{yr}$, while $1986 \mathrm{~J}$ was at least 20 $M_{o}$ and had a somewhat stronger wind. Recent VLBI data show the growing remnant to be somewhat elongated.

Late-time spectroscopy of 1988Z, a peculiar SN II (E. Sadler). These results have now been published by R.A. Stathakis and E.M. Sadler (1991, MNRAS 250, 786).

Supernova remnants observed by ROSAT (B. Aschenbach). The ROSAT survey has resulted in the first SNRs to be discovered first as X-ray sources; the complete sky is expected to contain about 60 or these. $1987 \mathrm{~A}$ has not yet been detected, down to a level of $2.5 \times 10^{34} \mathrm{erg} / \mathrm{s}$. Magnificent images have been produced for RCW 103, SNR 18.9-1.1, and other remnants.

Supernova remnants observed by BBXRT (R. Petre). A scan of the LMC region has not detected SN $1987 \mathrm{~A}$, somewhat below the ROSAT limit and considerably below the flux reported by Ginga in 1988 . The spectrum of N132D reveals lines of $\mathrm{O}, \mathrm{Mg}, \mathrm{Si}, \mathrm{S}$, and $\mathrm{Fe}$, in relatively high ionization states. An abundance analysis shows that this thousand-year-old remnant must consist largely of swept up interstellar matter, since the metallicity is about 0.2 that of the sun, the same as the general ISM around it. [Fe/O] is negative, as expected for metal-deficient material.

Supernova remnants observed by ASTRO (T. Stecher). Five SNRs were scanned with this ultraviolet experiment, yielding a two-sigma detection of 1987A and images of the Crab Nebula that resemble those in the higher-excitation optical lines.

The Cygnus superbubble (M. Shull). IRAS maps of this recent star forming region show a superbubble in the process of formation, with dust at $30-35 \mathrm{~K}$ and energy input from the $\mathrm{O}$ stars of Cyg OB1 as well 
as one or two Wolf Rayet stars and (probably) 5-10 supernovae. The stars of Cyg OB2 have also contributed to the superbubble of the Cygnus $X$ region revealed in the ROSAT images.

\section{Hamuy: The Optical Light Curves of Type Ia Supernovae 1980N and 1981D in Fornax A.}

We present optical photometry of the two supernovae, $1980 \mathrm{~N}$ and 1981D, which appeared in the peculiar D-type galaxy NGC 1316 (Fornax A). These data are combined with published observations to produce definitive optical light curves. We find that the maximum-light magnitudes of both supernovae were the same to within $\pm 0.1 \mathrm{mag}$, in agreement with infrared light curve observations. The shapes of the UBV light curves of the best observed of the two supernovae, 1980N, closely resembled those of the type Ia prototype SN 1981B. We also show that an optical spectrum of SN $1980 \mathrm{~N}$ taken 30 days after B maximum was virtually identical to a spectrum of SN 1981B obtained at the same point in its evolution. These findings lend support to claims that the majority of type Ia supernovae form a highly homogeneous class of objects. Nevertheless, the B-V colors at B maximum of the NGC 1316 supernovae were 0.3-0.5 mag redder than previous estimates of the intrinsic B-V color of type Ia supernovae at this phase. Although dust extinction within NGC 1316 could explain this difference, there is little evidence to support such a large reddening. By comparing the photometric data of SN 1980N with the light curves of SN 1984A in NGC 4419, and by assuming that the absolute magnitudes of the majority of type Ia supernovae are indeed very similar, NGC 1316 would appear to be at essentially the same distance as the core of the Virgo Cluster.

\section{Cecilia Kozma and Claes Fransson: Radioactive Excitation in SN 1987A and other Supernovae.}

We have calculated the gamma-ray deposition in different composition zones in core collapse supernovae using the Spencer-Fano equation. For these results we have given analytical expressions. The same calculations have been done for pure helium, oxygen, and iron plasmas. We have also calculated the fraction of the absorbed gamma-ray energy which is re-emitted as photons with energy about $3.4 \mathrm{eV}$, the $\mathrm{n}$ $=2$ threshold for hydrogen. We have applied these results to calculate the H-alpha luminosity from SN $1987 \mathrm{~A}$, and we find good agreement with observations.

\section{P. Lundqvist: Narrow Lines from SN 1987A.}

New models, based on a ring geometry, have been used to model the narrow line emission from SN $1987 \mathrm{~A}$. With a tilt angle of 45 degrees for the ring, the ring radius is $\sim 6 \times 10 \mathrm{E} 17 \mathrm{~cm}$. Gas density, elemental composition and properties of the ionizing source are close to what has previously been found for a shell geometry (1). The most important improvement compared to the old models, is that ring models may be optically thick to the ionizing burst. This results in better agreement with observations for low-ionization lines like N III] 1750 and N IV] 1486. An improved treatment for the diffuse emission, as well as escape probabilities gives more [O III] emission at late times, also in agreement with observations. Finally, it is noted that several lines should be observable, especially lines of Fe II and Fe III. It is likely that a recently observed line at $5159 \mathrm{~A}$, and which was identified as a Fe VII line (2), is instead a Fe II line. From calculated line intensities [Fe II] 5159 is expected to be as strong as observed Fe II lines at $7155 \mathrm{~A}(2,3)$ and 8617A (2).

(1) Lundqvist, P., and Fransson, C., 1991, ApJ, in press. (2) Wang, L., 1991, preprint. (3) Meikle, P., and Cumming R., 1991, priv.comm.

\section{July: WG on Supernovae}

CHAIR: W. Liller

I. SN 1987A Update (total time $=30 \mathrm{~min}$.)

Suntzeff, N.B., CTIO. Bolometric light curves.

Bouchet, P., ESO/La Silla. ESO observations.

Meikle, W.P., Imperial College London. IR spectroscopy. 
II. Current Status of our Understanding of SNe $(60 \mathrm{~min}$.

Phillips, M.M., CTIO. Optical spectra.

Dubner, G., IAFE. VLA observations of SNe.

Maza, J., U. of Chile. SN Searches and results.

Van den Bergh, S., DAO. Rates, distribution.

Wheeler, J.C., U. Texas. Theoretical spectra.

Chugai, N.N., U.Moscow. Physics of Type II SNe.

Nicholas B. Suntzeff, M.M. Phillips, D.L. Depoy, J.H. Elias, and A.R. Walker: The Late-Time Bolometric Evolution of SN 1987A.

We review our results on the optical, infrared, and bolometric luminosity evolution of SN 1987A through day 1444 since the explosion of the supernova. We find that both the optical and mid-infrared magnitudes are slowly leveling out after their rapid decline in brightness during the epoch of dust formation. The optical magnitudes are declining with an e-folding time of near 400 days, which is quite close to the rate expected from the decay of ${ }^{57} \mathrm{Co}$. The near infrared colors initially reddened after dust formation around day 500 , but by day 1000 , they began to evolve to the blue, perhaps due to the thinning of the dust. Optical spectra obtained through day 1500 show no evidence for a sudden drop in the optical emission-line luminosities. This suggests that the region of optical emission has not undergone the so-called "infrared catastrophe", when the cooling of the ejecta becomes dominated by the infrared fine-structure lines, at least in the case described by the simple models of Fransson and Chevalier (1987).

The infrared data have been combined with optical photoelectric and CCD UBVRI photometry obtained at CTIO to study the temporal evolution of the bolometric luminosity of SN 1987A. By day 1000, another source of energy beside the radioactive decay of ${ }^{56} \mathrm{Co}$ is needed to explain the slow leveling off of the bolometric luminosity decline. This extra source of energy must be declining in time, and a constant energy source such as ${ }^{44} \mathrm{Ti}$ can be ruled out. The best fit to the data is for $5 \pm 1$ times the predicted ("solar") amount of ${ }^{57} \mathrm{Co} /{ }^{56} \mathrm{Co}$. If a pulsar or other compact energy source is present, the energy input must have declined from $\sim 37.5$ dex $\left(\log _{10}\left(\mathrm{erg} \mathrm{s}^{-1}\right)\right)$ on day 1000 to $\sim 36.8$ dex on day 1444 . The enhanced ${ }^{57} \mathrm{Co}$ which provides the best fit to the data is in conflict with published hard $x$-ray and infrared spectral data. However, a careful reanalysis of the uncertainties in the observed hard X-ray and uvoir fluxes, and the model predictions, suggests that all the data can be fit by the energy deposition from $0.075 \mathrm{M}_{\mathrm{O}}$ of ${ }^{56} \mathrm{Ni}$, a ${ }^{57} \mathrm{Co} /$ ${ }^{56} \mathrm{Co}$ between 2.5 and 4 times "solar", and "solar" ${ }^{44} \mathrm{Ti}$.

P. Bouchet, I.J. Danziger, C. Gouiffes, L.B. Lucy and M. Della Valle - SN 1987A: Dust and line luminosities: Observations of the later phases at ESO.

We review the evidence for dust formation and its spectral characteristics. We show that dust is still present and is absorbing more strongly than ever at least up till day 1400 . We present a simplified qualitative model of the expanding envelope with 2 zones: a higher velocity hotter region, and a lower velocity cooler region where the IR catastrophe could have started at day $\sim 760$. Our observations exclude a value of the ${ }^{56} \mathrm{Co} /{ }^{57} \mathrm{Co}$ ratio as high as $4 \mathrm{x}$ solar. Then, the possible source of energy required to give account to the observed flattening of the bolometric light curve is probably a compact central object, radiating as a pulsar, or surrounded by an accretion disk depositing matter either continuously or at varying intervals onto the collapsed object.

The presence of such a source of energy is also required to keep the gas in the circumstellar ring surrounding SN 1987A excited, as we show that the excitation of this material cannot be due solely to the initial UV outburst.

Peter Meible: IR Spectroscopy of SN 1987A - an update.

The introduction of more sensitive near infrared detectors has meant that we can still observe SN 1987A spectroscopically. Using the new infrared facility, IRIS, at the AAT, we have extended our spectroscopic coverage to around day 1500 (after the explosion). The spectra cover 0.9 to $2-4 \mu \mathrm{m}$ at a resolution of 400 and aperture of $5^{\prime \prime} \times 12^{\prime \prime}$. Circumstellar lines are increasingly prominent, especially $\mathrm{H} \alpha \mathrm{I} 1.083 \mu \mathrm{m}$. Resolved features arising from the ejecta can still be seen. Ejecta [Fe II] lines have faded significantly rel- 
ative to other features, over the last $\sim 500$ days. This may be due to recombination of $\mathrm{Fe}^{+}$to $\mathrm{Fe}^{\mathrm{o}}$. [Fe I] lines are still prominent.

Since about day 700 , lines of $\mathrm{HI}, \mathrm{Na}$ I, Si I have matched the radioactive luminosity decay. This suggests that there has been no increase in the transparency of the emission regions to the $\chi$-rays over a doubling of the supernova age. Exploration of this may require the persistence of clumps which are optically thick to the $\chi$-rays.

Preliminary modeling of the IR spectra (based on model $10 \mathrm{HM}$ abundances) confirms more line identifications.

\section{M.M. Phillips: Optical Spectroscopy of Type I Supernovae.}

Optical spectroscopic observations of several recent type I supernovae are presented. The type Ia event $1991 \mathrm{~T}$ is of particular interest since the first spectra, obtained approximately one week before maximum, did not show any trace of the $\mathrm{Si}$ II $\lambda 6355$ and $\mathrm{Ca} \mathrm{II} \mathrm{H} \mathrm{k} \mathrm{K}$ absorption which normally characterizes these early phases. These lines finally did appear around maximum light, and by ten days after maximum the spectrum of SN $1991 \mathrm{~T}$ had evolved to closely resemble the spectra of other well-observed type Ia supernovae at comparable epoches. These data provide dramatic evidence that type Ia supernovae are not a perfectly homogeneous class of objects. The spectral differences observed for SN $1991 \mathrm{~T}$ suggest that the distribution of intermediate mass elements in the outer layers of the ejecta of these objects can differ significantly from one event to another.

Spectra of several recent type $\mathrm{Ib}$ (helium-rich) and Ic (helium-poor) supernovae are also presented. These objects show a fairly large dispersion in relative line strengths and widths. Indeed, evidence is found for a continuous range of helium line strengths, which suggests that the $\mathrm{Ib}$ and Ic designations are actually the extremes of a single class of supernovae.

\section{G. Dubner ${ }^{(1)}, M$. Goss $^{(2)}$, F. Winkler ${ }^{(3)}$ and D. Moffett $t^{(2)}$; VLA Observations of Supernova Remnants. \\ (1) IAFE, Buenos Aires, Argentina; (2) NRAO, Socorro, USA; (3) Middlebury College, Vermont, USA}

We report radio continuum observations of 14 catalogued galactic SNRs located very close to the galactic plane, between $\imath=-10^{\circ}$ and $\imath=+47^{\circ}$. The observations were done in 1990 and 1991 with the VLA in the $\mathrm{D}$ and $\mathrm{C} / \mathrm{D}$ configurations, providing at $1465 \mathrm{MHz}$ an angular resolution of about $50^{\prime \prime}$ and 35 " respectively, with an average noise of about $6 \mathrm{mJy} / \mathrm{beam}$. Two out of 14 sources were found to show a "compositetype" morphology. Other six remnants appear as almost complete circular shells; the rest are incomplete shells with the visible portion aligned with the galactic plane.

\section{José Maza: Supernova Searches and Results.}

A total of $766 \mathrm{SNe}$ have been detected since S Andromedae in 1885 . Of those 714 have been discovered since 1950. The number of SNe per year have increased from an average of 10 in 1950 up to 20 in 1985 . In $1991 \mathrm{SN}$ searches have produced a record of 37 in half a year! From 1979 to 1984 a total of 46 SNe were discovered at Cerro El Roble (University of Chile), $43 \%$ of the total number discovered in the world during that period. Now a new program of SN search has been started in 1990, involving Cerro Tololo Interamerican Observatory and the University of Chile. We have detected 9 new SNe in 10 months.

\section{S. van den Bergh: The Galactic Supernova Rate.}

Historical observations yield a "local" supernova rate of 5 per millennium within $3 \mathrm{kpc}$ of +4 sun. This value is much greater than that calculated from various indirect techniques (see table).

Method

Historical $(\mathrm{R}<3 \mathrm{kpc})$

Stellar LF new Sun

Comparison with LMC WR Stars
SNe/1000 yr

5

$\sim 0.3$

$0.2 \pm 0.1$ 
Gas depletion timescale 5 Gyr. Shapley-Ames galaxies
0.2

$(2.2 \pm 1.0) h^{2}$

The "historical" SN rate would correspond to a total Galactic supernova rate of 15-20 per century. Or put in another way it would imply that the SN rate in all of M33 is lower than that in the region within $3 \mathrm{kpc}$ of the Sun.

No plausible explanation for this discrepancy has been found.

\section{J. Craig Wheeler: Theoretical Spectra of Supernovae.}

Atmosphere models of Type Ia supernovae near maximum light show that Nomoto's carbon deflagration model provides an especially good agreement with observations compared to other similar models. The same model does not agree as well with early spectra of SN 1990N, but suggests that the absorption feature at $3200 \mathrm{~A}$ is not radioactive cobalt, but a blend of titanium lines.

Observations of the nebular spectra of SN 1987A at 2200 days are consistent with a significant contribution to the emission lines coming from the solar abundance of heavy elements in the outer envelope. The helium in the envelope is sufficient to give a strong line of He I $\lambda$ 10830. Until the core and envelope contributions are adequately deconvolved, it will be difficult to determine the quantity of freshly synthesized oxygen.

Models for Type $\mathrm{Ib} / \mathrm{c}$ supernovae have been computed based on helium cores of moderately massive stars. Such models are too cool near maximum and overly contaminated by Fe II. Models of the nebular phase suggest that the optical spectrum is rather insensitive to significant changes in the composition but that He $I \lambda 10830$ is a key diagnostic of the helium abundance.

\section{N.N. Chugai: SNe II Powered by Ejecta-wind Interactions.}

The interaction of the expanding ejecta of the type II supernova with the pre-SN wind may contribute noticeably to the optical output of SN II, particularly at late times. The strong excess of the $\mathrm{H} \alpha$ flux over the model of the radioactive source is revealed in the late-time behavior of SNe II 1979C, $1980 \mathrm{~K}$, and 1987F. The bulk of observational properties strongly suggests that this excess is energized by the ejecta-wind interaction. Furthermore, the extremely high luminosity of SN 1987F (as well as its counterpart, SN 1988Z) is probably also powered by the interaction of the ejecta with the very dense wind $\left(\mathrm{M} / \mathrm{u}_{\mathrm{w}} \sim 10^{17} \mathrm{~g} \mathrm{~cm}^{-1}\right)$. 